Tropical Journal of Pharmaceutical Research January 2021; 20 (1): 23-28

ISSN: $1596-5996$ (print); 1596-9827 (electronic)

(C) Pharmacotherapy Group, Faculty of Pharmacy, University of Benin, Benin City, 300001 Nigeria.

\title{
Downregulation of FOXO4 promotes neuronal survival by mediating oxidative-stress-induced apoptosis after cerebral ischemia/reperfusion injury
}

\author{
Chunying Deng ${ }^{1,2}$, Peilan Zhang ${ }^{1 *}$, Yun Zhang ${ }^{1,3}$ \\ ${ }^{1}$ Department of Neurology, Tianjin Huanhu Hospital, Tianjin 300350; ${ }^{2}$ Department of Neurology, North China University of \\ Science and Technology Affiliated Hospital; ${ }^{3}$ Department of Geriatrics, Tangshan Gongren Hospital, Tangshan City, Hebei \\ Province 063000, China
}

*For correspondence: Email: zhangpeilan666@163.com; Tel: +86-022-59065241

Sent for review: 13 October 2020

Revised accepted: 28 December 2020

\begin{abstract}
Purpose: To investigate the effect of FOXO4 on cerebral ischemia/reperfusion (CIR) injury and the underlying mechanism.

Methods: An in vitro ischemia/reperfusion (IR) model was achieved using oxygen-glucose deprivation/reoxygenation (OGD/R). Expression of RNA and protein was determined using quantitative real time polymerase chain reaction ( $(R R T-P C R)$ and western blotting, respectively. Cell viability and apoptosis were determined using MTT assay and flow cytometry, respectively. Commercial kits were used to measure lactate dehydrogenase ( $L D H)$, reactive oxygen species (ROS), chloramphenicol acetyltransferase (CAT), malondialdehyde (MDA), and superoxide dismutase (SOD).

Results: Following OGD/R, FOXO4 mRNA and protein expressions were upregulated in SH-SY5H human neuroblastoma cells. ODG/R reduced cell proliferation and increased the proportion of apoptotic cells, and these effects were inhibited by knockdown of FOXO4 $(p<0.05)$. Levels of cleaved caspase 3 and cleaved poly(ADP-ribose) polymerases (PARPs) were increased after ODG/R and these increases were inhibited by FOXO4 knockdown. ROS content and levels of $L D H$ and MDA were increased after $O D G / R$ and decreased by knockdown of FOXO4 $(p<0.05)$. Levels of CAT and SOD were reduced after $O D G / R$, and this reduction was reversed by knockdown of FOXO4 $(p<0.05)$.

Conclusion: The results demonstrate that knockdown of FOXO4 promotes cell proliferation and inhibits cellular apoptosis via reduction of oxidative stress after CIR injury, indicating a new therapeutic target for the treatment of CIR injury.
\end{abstract}

Keywords: FOXO4, Neuronal survival, Oxidative stress, Cerebral ischemia/reperfusion injury

This is an Open Access article that uses a fund-ing model which does not charge readers or their institutions for access and distributed under the terms of the Creative Commons Attribution License (http://creativecommons.org/licenses/by/4.0) and the Budapest Open Access Initiative (http://www.budapestopenaccessinitiative.org/read), which permit unrestricted use, distribution, and reproduction in any medium, provided the original work is properly credited.

Tropical Journal of Pharmaceutical Research is indexed by Science Citation Index (SciSearch), Scopus, International Pharmaceutical Abstract, Chemical Abstracts, Embase, Index Copernicus, EBSCO, African Index Medicus, JournalSeek, Journal Citation Reports/Science Edition, Directory of Open Access Journals (DOAJ), African Journal Online, Bioline International, Open-J-Gate and Pharmacy Abstracts

\section{INTRODUCTION}

Cerebral ischemia/reperfusion occurs when blood supply to suspended and subsequently
(CIR) injury the brain is restored [1].
Cerebral IR injury induces brain dysfunction, which contributes to high mortality and disability $[2,3]$. According to the World Health Organization (WHO), 15 million strokes occur worldwide each year, with more than 6 million deaths and 
another 5 million disabled [4]. The mechanism of CIR injury is complicated and unclear. Thus, the pathological process of CIR injury should be investigated to understand and treat CIR injury in the future.

Many biological processes are involved in CIR injury. Oxidative stress has been reported to occur in almost all cases of CIR injury, resulting in excessive production of reactive oxygen species (ROS) [5,6]. Excessive ROS may cause cell death and tissue necrosis by inducing DNA damage, lipid peroxidation, cytoskeletal structural injury, and chemotaxis [5]. Poly(ADP-ribose) polymerases (PARPs) are a class of zinc-finger DNA-binding proteins that are essential for cellular response to DNA damage [7]. Among the PARPs, PARP-1 has been reported to regulate inflammation in central nervous system disorders, including CIR injury [8]. Overactivation of PARP-1 has been detected in the brain after brain ischemia and cardiac arrest in response to oxidative DNA damage [9]. Ischemic injury was prevented in a PARP knockout mouse model compared with wild-type mice, indicating that inhibition of PARP is a potential new therapeutic strategy for CIR injury [10].

FOXO4 is a member of the Forkhead (Fox) transcription factor $O$ family, which regulates multiple pathophysiological processes, including cellular apoptosis, oxidative stress, and cell cycle arrest [11]. In a rat model of myocardial IR injury, upregulation of $\mathrm{FOXO} 4$ promoted myocardial apoptosis through increasing ROS generation [12]. During renal IR injury, production of hydrogen peroxide was induced through overexpression of $\mathrm{FOXO4}$, resulting in renal cell apoptosis in mice [13]. However, there are no data on the role of FOXO4 in CIR injury. Thus, the purpose of this study was to investigate the effects of FOXO4 on CIR injury and provide a new treatment target for CIR injury.

\section{EXPERIMENTAL}

\section{Cell culture}

SH-SY5H human neuroblastoma cells were obtained from the American Type Culture Collection (ATCC, Manassas, VA, USA) and cultured in Dulbecco's modified Eagle medium (DMEM) supplemented with $10 \%$ fetal bovine serum (FBS, Gibco, Grand Island, NY, USA), Glutamax (Gibco), 100 units/ml penicillin/streptomycin (Invitrogen, Carlsbad, CA, USA), and 1× MEM non-essential amino acids (Gibco) in a humidified atmosphere of $5 \% \mathrm{CO}_{2}$ at $37^{\circ} \mathrm{C}$.
The in vitro IR model was achieved by oxygenglucose deprivation/reoxygenation (OGD/R) [14]. Briefly, the SH-SY5H cells were seeded in 12well plates at a density of $1 \times 10^{5}$ cells/well in glucose-free DMEM with $1 \% \mathrm{O}_{2}, 94 \% \mathrm{~N}_{2}$, and $5 \% \mathrm{CO}_{2}$ (hypoxic conditions) and incubated at $37^{\circ} \mathrm{C}$ for $3 \mathrm{~h}$. The medium was changed to standard DMEM with $95 \% \mathrm{O}_{2}$ and $5 \quad \% \quad \mathrm{CO}_{2}$ (normoxic conditions) and cells were incubated for up to $48 \mathrm{~h}$. SH-SY5H cells cultured in standard medium under normoxic conditions were used as the control group.

Small hairpin RNA (shRNA) targeting FOXO4 (shFOXO4) and negative control shRNA (shNC, Ibsbio, China) were mixed with Lipofectamine 2000 (Invitrogen) according to manufacturer's protocols. The $\mathrm{SH}-\mathrm{SY} 5 \mathrm{H}$ cells were cultured in 6well plates at a density of $1 \times 10^{6}$ cells/well in serum-free medium. The transfection mixture was added to the cells and incubated for $6-8 \mathrm{~h}$. The medium was then changed to standard culture medium. SH-SY5H cells were cultured for $24 \mathrm{~h}$ at $37^{\circ} \mathrm{C}$ and collected for further analysis.

\section{MTT assay}

The SH-SY5H cells were cultured in 96-well plates. After transfection, MTT $(5 \mathrm{mg} / \mathrm{ml}$ in PBS; Abcam, Cambridge, UK) was added and incubated for $4 \mathrm{~h}$ according to the manufacturer's instructions. The formazan was dissolved using dimethyl sulfoxide. The absorbance value at 490 $\mathrm{nm}$ was measured using a spectrophotometer (BioTek, Winooski, VT, USA).

\section{Extraction of RNA and quantitative (real-time) PCR (qPCR)}

Total RNA extraction was performed using TRIzol reagent (Thermo Fisher Scientific Waltham, MA, USA). Reverse transcription was performed with $1 \mu \mathrm{g}$ RNA using the QuantiTect Reverse Transcription Kit (QIAGEN, Hilden, Germany). RNA expression level was measured using the QuantiTect SYBR Green RT-PCR Kit (QIAGEN) with the StepOnePlus system (Applied Biosystems, Foster City, CA, USA). Relative RNA expression was quantified using the $2^{-\Delta \Delta C t}$ method [15]. Sequences of primers (SigmaAldrich, St. Louis, MO, USA) used in this study are shown in Table 1.

\section{Western blotting}

Cell lysates were prepared by extraction of cells in RIPA cell lysis buffer (Beyotime, Shanghai, China). Proteins were separated through a $7.5 \%$ SDS-PAGE gel. The separated proteins were then transferred to PVDF membranes, followed 
by blocking of membranes with $5 \%$ fat-free milk. The blocked membranes were probed with the appropriate primary antibody overnight at $4{ }^{\circ} \mathrm{C}$ and then incubated with secondary antibodies for $2 \mathrm{~h}$ at room temperature. Protein bands were detected using ECL Detection reagents (SigmaAldrich). The primary antibodies used in this study (all from Cell Signaling Technology, Danvers, MA, USA) were anti-FOXO4 (\#9472, 1:1000 dilution), anti-cleaved caspase 3 (\#9664, 1:1000 dilution), anti-cleaved PARP (\#5625, 1:1500 dilution), anti- $\beta$-actin (\#4970, 1:5000 dilution), and anti-GAPDH (\#5174, 1:5000 dilution).

Table 1: Sequences of primers used in this study

\begin{tabular}{|c|c|}
\hline $\begin{array}{l}\text { Gene } \\
\text { name }\end{array}$ & Primer sequence \\
\hline FOXO4 & $\begin{array}{l}\text { Forward: 5'- } \\
\text { GGCTGCCGCGATCATAGAC-3' } \\
\text { Reverse: 5'- } \\
\text { GGCTGGTTAGCGATCTCTGG-3' }\end{array}$ \\
\hline GAPDH & $\begin{array}{l}\text { Forward: 5'- } \\
\text { TGTGGGCATCAATGGATTTGG-3' } \\
\text { Reverse: 5'- } \\
\text { ACACCATGTATTCCGGGTCAAT-3' }\end{array}$ \\
\hline
\end{tabular}

\section{Flow cytometry}

After transfection with shRNA for $24 \mathrm{~h}, \mathrm{SH}-\mathrm{SY} 5 \mathrm{H}$ cells were collected and resuspended at a concentration of $1 \times 10^{7}$ cells $/ \mathrm{ml}$. The cell suspension was stained with Annexin-V-FITC and propidium iodide (Sigma-Aldrich) for 15 minutes. Apoptosis was detected using a FACS Calibur flow cytometer (BD Biosciences, San Jose, CA, USA). Cells $\left(2 \times 10^{4}\right)$ were collected, recorded, and the data were presented as twoparameter dot-plots.

\section{Measurement of biochemical parameters}

Lactate dehydrogenase (LDH), reactive oxygen species (ROS), catalase (CAT), malondialdehyde (MDA), and superoxide dismutase (SOD) were measured using the LDH assay kit (Abcam), ROS detection assay kit (Abcam), CAT assay kit (GenScript, Piscataway, NJ, USA), MDA assay kit (Abcam), and SOD activity assay kit (Abcam) according to the manufacturers' instruction.

\section{Statistical analysis}

GraphPad Prism 8.0 software (GraphPad, San Diego, CA, USA) was used to carry out all data analysis. Student's $t$-test was used to compare differences between two groups. One-way ANOVA was used to compare multiple groups. All data are expressed as mean \pm standard deviation (SD). $P<0.05$ was considered statistically significant.

\section{RESULTS}

\section{FOXO4 expression was upregulated by OGD/R in SH-SY5H cells}

After OGD/R, expression of FOXO4 mRNA was significantly upregulated in $\mathrm{SH}-5 \mathrm{Y} 5 \mathrm{H}$ cells compared with the control group (Figure $1 \mathrm{~A}$ ). FOXO4 protein was also upregulated after OGD/R (Figure $1 \mathrm{~B}$ ).
A

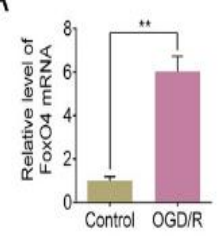

B

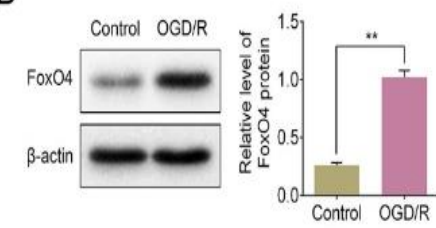

Figure 1: Expression of $\mathrm{FOXO} 4$ was upregulated after OGD/R in SH-SY5H cells. (A) Expression of FOXO4 mRNA was upregulated after OGD/R; (B) Expression of FOXO4 protein was upregulated after OGD/R; ${ }^{* *} p<$ 0.01 vs. control. OGD/R: oxygen-glucose deprivation/reoxygenation

\section{Knockdown of FOXO4 facilitated OGD/R-} induced survival of SH-SY5H cells

Expression of FOXO4 mRNA was decreased in $\mathrm{SH}-\mathrm{SY} 5 \mathrm{H}$ cells transfected with shFOXO4, compared with cells transfected with shNC in both control and OGD/R groups (Figure 2 A). Protein expression of FOXO4 was significantly downregulated in $\mathrm{SH}-\mathrm{SY} 5 \mathrm{H}$ cells transfected with shFOXO4, compared with cells transfected with shNC in both control and OGD/R groups (Figure $2 \mathrm{~B})$. Cell viability was increased in $\mathrm{SH}-\mathrm{SY} 5 \mathrm{H}$ cells transfected with shFOXO4, compared with cells transfected with shNC in the OGD/R group (Figure $2 \mathrm{C}$ ). In cells cultured in standard medium under normoxic conditions, there was no difference in viability between cells transfected with shFOXO4 and shNC (Figure $2 \mathrm{C}$ ). LDH activity did not show any significant change after transfection with shFOXO4 in cells cultured in standard medium under normoxic conditions (Figure 2 D). In the OGD/R group, LDH activity was decreased in cells transfected with shFOXO4, compared with its negative control group (Figure $2 \mathrm{D}$ ).

\section{Knockdown of FOXO4 prevented OGD/R- induced apoptosis in SH-SY5H cells}

The results of flow cytometric analysis demonstrated that OGD/R promoted apoptosis, 
compared with the control group, whereas downregulation of $\mathrm{FOXO4}$ reduced the proportion of apoptotic cells induced by OGD/R (Figure $3 \mathrm{~A}$ ). Levels of cleaved caspase 3 and cleaved PARP were increased after OGD/R, whereas knockdown of $\mathrm{FOXO} 4$ reduced the increased levels of cleaved caspase 3 and cleaved PARP induced by OGD/R (Figure $3 \mathrm{~B}$ ).

A

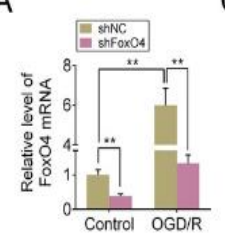

C
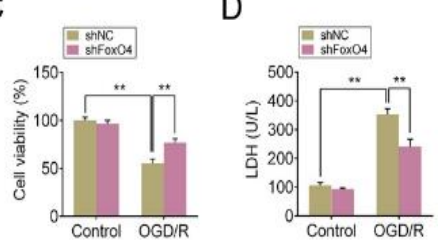

B
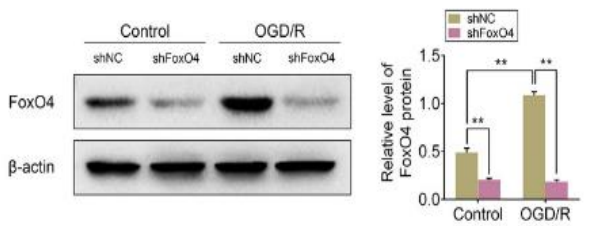

Figure 2: Knockdown of FOXO4 facilitated OGD/Rinduced cell survival in $\mathrm{SH}-\mathrm{SY} 5 \mathrm{H}$ cells. (A) shFOXO4 reduced the upregulation of FOXO4 mRNA induced by $\mathrm{OGD/R}$; (B) shFOXO4 reduced the upregulation of FOXO4 protein induced by OGD/R; (C) shFOXO4 promoted cell survival after OGD/R; (D) shFOXO4 reduced the increase in LDH induced by OGD/R; ${ }^{* *} p<$ 0.01 vs. control or shNC. LDH: lactate dehydrogenase; OGD/R: oxygen-glucose deprivation/reoxygenation; shFOXO4: short hairpin RNA (shRNA) targeting FOXO4; shNC: negative control shRNA
A
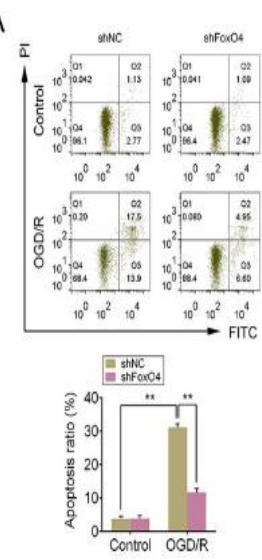

B
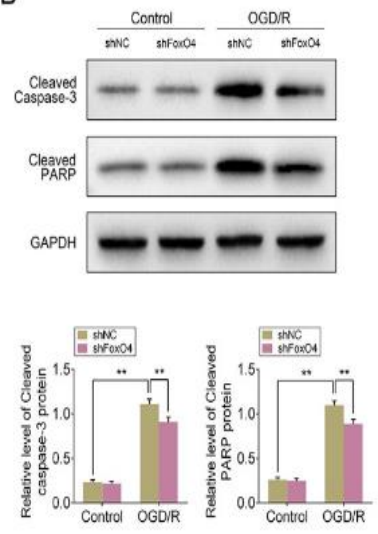

Figure 3: Knockdown of FOXO4 prevented OGD/Rinduced apoptosis in SH-SY5H cells. (A) shFOXO4 prevented apoptosis induced by OGD/R; (B) shFOXO4 inhibited the increase in levels of cleaved caspase 3 and cleaved PARP induced by OGD/R; ${ }^{* *} p$ $<0.01$ vs. control or shNC. OGD/R: oxygen-glucose deprivation/reoxygenation; PARP: poly(ADP-ribose) polymerase; shFOXO4: short hairpin RNA (shRNA) targeting FOXO4; shNC: negative control shRNA
Knockdown of FOXO4 reduced OGD/Rinduced oxidative stress in SH-SY5H cells

After OGD/R, ROS content was significantly increased, whereas the increase in ROS content was inhibited by knockdown of FOXO4 (Figure 4 A). CAT activity was reduced after OGD/R and this reduction was suppressed by knockdown of FOXO4 (Figure 4 B). After OGD/R, MDA was significantly increased, whereas this increase was inhibited by knockdown of FOXO4 (Figure 4 C). SOD activity was reduced after OGD/R and this reduction was reversed by shFOXO4induced downregulation of FOXO4 (Figure $4 \mathrm{D}$ ).
A

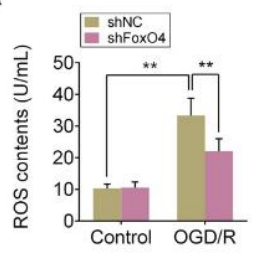

C

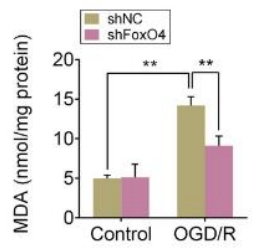

B

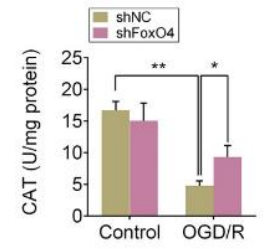

D

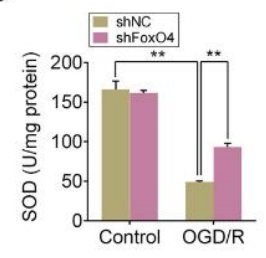

Figure 4: Knockdown of FOXO4 reduced OGD/Rinduced oxidative stress in $\mathrm{SH}-\mathrm{SY} 5 \mathrm{H}$ cells. (A) shFOXO4 inhibited the increase in ROS content induced by OGD/R; (B) shFOXO4 prevented the inhibition of MDA induced by OGD/R; (C) shFOXO4 inhibited the increase in MDA induced by OGD/R; (D) shFOXO4 prevented the inhibition of SOD induced by $\mathrm{OGD} / \mathrm{R} ;{ }^{* *} \mathrm{p}<0.01$ vs. control or shNC. CAT: chloramphenicol acetyltransferase; MDA: malondialdehyde; OGD/R: oxygen-glucose deprivation/reoxygenation; ROS: reactive oxygen species; shFOXO4: short hairpin RNA (shRNA) targeting FOXO4; shNC: negative control shRNA; SOD: superoxide dismutase

\section{DISCUSSION}

OGD is a common in vitro IR model that is usually used to investigate ischemia-induced cellular dysfunction [16]. Sustained OGD $(>1 \mathrm{~h})$ followed by re-oxygenation (OGD/R) contributes to mitochondrial dysfunction, ROS production, and cellular necrosis (but not apoptosis) [16]. In this study, expression of FOXO4 mRNA and protein was upregulated in the $O G D / R$ neuroblastoma cells. Further exploration of the mechanism underlying this effect demonstrated that knockdown of FOXO4 inhibits OGD/Rinduced cell proliferation and ROS production and promotes apoptosis, indicating that inhibition

Trop J Pharm Res, January 2021; 20(1): 26 
of $\mathrm{FOXO} 4$ is a potential new therapeutic target for CIR injury.

As mentioned above, FOXO4 regulated $\mathrm{ROS}$ generation after myocardial IR injury and renal IR injury [12,13]. In this study, ROS content was increased after $O G D / R$, and downregulation of FOXO4 reduced OGD/R-induced ROS production, indicating that $\mathrm{FOXO} 4$ controlled ROS generation in CIR injury, which is consistent with observations in myocardial IR injury and renal IR injury. SODs are a class of enzymes catalyzing the conversion of superoxide into oxygen and hydrogen peroxide and poison of ROS [17]. MDA has been widely used as a biomarker of oxidative stress [18].

In this study, SOD activity was reduced, and MDA was increased after OGD/R in neuroblastoma cells; inhibition of FOXO4 expression prevented this phenomenon, suggesting that upregulation of FOXO4 promoted an increase in oxidative stress during CIR injury. In addition, it is well known that oxidative stress is associated with cellular apoptosis [19]. Overproduction of ROS induced neuronal cell damage through an apoptotic or a necrotic pathway, promoting cell death [20]. The results of this study demonstrated that cell viability was reduced after $\mathrm{OGD} / \mathrm{R}$ and negatively correlated with FOXO4 expression, indicating that overexpression of FOXO4 accelerated apoptosis after CIR injury. Taken together, the data show that in this in vitro CIR model, upregulation of FOXO4 contributed to oxidative stress-induced apoptosis in neuroblastoma cells. Inhibition of FOXO4 demonstrated the protective effects on neuroblastoma cells, implying a new therapeutic strategy for CIR injury.

Caspase 3 is a crucial enzyme in the process of programmed cell death (PCD), such as in apoptosis and pyrosis [21]. Caspase 3 is activated through cleavage by upstream caspases and translocated from the cytoplasm into the nucleus in cells undergoing PCD [21]. In this study, cleaved caspase 3 increased after $\mathrm{OGD} / \mathrm{R}$, and knockdown of FOXO4 reduced the level of increased cleaved caspase 3, indicating that FOXO4 mediated PCD in CIR injury. PARP1 is a major substrate of caspase 3 [21]. Caspase 3 cleaves PARP-1 at position 214/215 to generate the $85-\mathrm{kDa}$ fragment in hepatocytes undergoing apoptosis [22]. In this study, cleaved PARP levels were increased by OGD/R and decreased by FOXO4 knockdown. This change in the level of cleaved PARP coincided with the change in cleaved caspase 3, further suggesting that inhibition of $\mathrm{FOXO} 4$ reduced apoptosis in CIR injury.

\section{CONCLUSION}

The findings of this study demonstrate that FOXO4 is upregulated after ODG/R. This upregulation of $\mathrm{FOXO} 4$ promotes apoptosis and inhibits cell proliferation, whereas FOXO4 knockdown inhibits apoptosis induced by oxidative stress after CIR injury, providing a new therapeutic target for CIR injury.

\section{DECLARATIONS}

\section{Acknowledgement}

This work was supported by Key R \& D SelfFinancing Projects in Hebei Province (Grant no. 172777151).

\section{Competing interests}

There is no conflict of interest to disclose.

\section{Authors' contributions}

We declare that this work was done by the authors named in this article and all liabilities pertaining to claims relating to the content of this article will be borne by the authors. Chunying Deng designed the study, supervised the data collection, and analyzed the data. Peilan Zhang interpreted the data and prepared the manuscript for publication. Yun Zhang supervised the data collection, analyzed the data, and reviewed the draft of the manuscript. All authors have read and approved the manuscript.

\section{Open Access}

This is an Open Access article that uses a funding model which does not charge readers or their institutions for access and distributed under the terms of the Creative Commons Attribution License (http://creativecommons.org/licenses/by/ 4.0) and the Budapest Open Access Initiative (http://www.budapestopenaccessinitiative.org/rea d), which permit unrestricted use, distribution, and reproduction in any medium, provided the original work is properly credited.

\section{REFERENCES}

1. Turley KR, Toledo-Pereyra LH, Kothari RU. Molecular Mechanisms in the Pathogenesis and Treatment of Acute Ischemic Stroke. J Invest Surg 2005; 18(4): 207218.

2. Yang J, Chen M, Cao RY, Li Q, Zhu F. The Role of Circular RNAs in Cerebral Ischemic Diseases: Ischemic 
Stroke and Cerebral Ischemia/Reperfusion Injury. Adv Exp Med Biol 2018; 1087: 309-325.

3. He L, He R, Liang R, Li Y, Li X, Li C, Zhang S. Protein expression profiling in the hippocampus after focal cerebral ischemia injury in rats. J Integr Neurosci 2018; 17(2): 277-292.

4. Rodrigo R, Fernández-Gajardo R, Gutiérrez R, Matamala $J M$, Carrasco R, Miranda-Merchak A, Feuerhake W. Oxidative stress and pathophysiology of ischemic stroke: novel therapeutic opportunities. CNS Neurol Disord Drug Targets 2013; 12(5): 698-714.

5. Khoshnam SE, Winlow W, Farzaneh M, Farbood Y, Moghaddam HF. Pathogenic mechanisms following ischemic stroke. Neurol Sci 2017; 38(7): 1167-1186.

6. Wang F, Wu L, Liang Q. Trigonoside II mitigates sepsisinduced myocardial injury via reduction in oxidative stress and regulation of TLR-4/NF-k $\beta$ inflammatory pathway. Trop J Pharm Res 2020; 19: 1161-1166.

7. Pascal JM. The comings and goings of PARP-1 in response to DNA damage. DNA Repair 2018; 71: 177182.

8. Castri P, Lee Y-J, Ponzio T, Maric D, Spatz M, Bembry J, Hallenbeck J. Poly(ADP-ribose) polymerase-1 and its cleavage products differentially modulate cellular protection through NF-kappaB-dependent signaling. BBA-Proteins Proteom 2014; 1843(3): 640-651.

9. Love $S$, Barber R, Wilcock GK. Neuronal accumulation of poly(ADP-ribose) after brain ischaemia. Neuropathol Appl Neurobiol 1999; 25(2): 98-103.

10. Endres M, Wang ZQ, Namura S, Waeber C, Moskowitz MA. Ischemic brain injury is mediated by the activation of poly(ADP-ribose)polymerase. J Cereb Blood Flow Metab 1997; 17(11): 1143-1151.

11. Liu W, Li Y, Luo B. Current perspective on the regulation of FOXO4 and its role in disease progression. Cellular and molecular life sciences: CMLS 2020; 77(4): 651663.

12. Yu L, Zhang $W$, Huang $C$, Liang $Q$, Bao H, Gong Z, Xu $M$, Wang $Z$, Wen $M$, Cheng $X$. FoxO4 promotes myocardial ischemia-reperfusion injury: the role of oxidative stress-induced apoptosis. Am J Transl Res 2018; 10(9): 2890-2900.

13. Liu H, Wang L, Weng X, Chen H, Du Y, Diao C, Chen Z, Liu $X$. Inhibition of Brd4 alleviates renal ischemia/reperfusion injury-induced apoptosis and endoplasmic reticulum stress by blocking FoxO4mediated oxidative stress. Redox biology 2019; 24: 101195.

14. Tiansong Yang DW, Yuanyuan Qu, Yulin Wang, Yuenan Feng, Yan Yang, Qiang Luo, Xiaowei Sun, Guoqiang Yu, Jia He, Zhongren Sun, Yulan Zhu. N-hydroxy-N'-(4butyl-2-methylphenyl)-formamidine attenuates oxygenglucose deprivation and reoxygenation-induced cerebral ischemia-reperfusion injury via regulation of microRNAs. J Integr Neurosci 2020; 19(2): 303-311.

15. Bubner B, Baldwin IT. Use of real-time PCR for determining copy number and zygosity in transgenic plants. Plant Cell Rep 2004; 23(5): 263-271.

16. Zheng $K$, Zhang Q, Lin G, Li Y, Sheng Z, Wang J, Chen L, Lu H-H. Activation of Akt by SC79 protects myocardiocytes from oxygen and glucose deprivation (OGD)/re-oxygenation. Oncotarget 2017; 8(9): 1497814987.

17. Wang Y, Branicky R, Noë A, Hekimi S. Superoxide dismutases: Dual roles in controlling ROS damage and regulating ROS signaling. J Cell Biol 2018; 217(6): 1915-1928.

18. Ayala A, Muñoz MF, Argüelles S. Lipid peroxidation: production, metabolism, and signaling mechanisms of malondialdehyde and 4-hydroxy-2-nonenal. Oxid Med Cell Longev 2014; 2014: 360438-360438.

19. Méndez-Armenta $M$, Nava-Ruíz $C$, Juárez-Rebollar $D$, Rodríguez-Martínez E, Gómez PY. Oxidative stress associated with neuronal apoptosis in experimental models of epilepsy. Oxid Med Cell Longev 2014; 2014 : 293689-293689.

20. Shin EJ, Jeong JH, Chung $Y H$, Kim WK, Ko KH, Bach JH, Hong JS, Yoneda Y, Kim HC. Role of oxidative stress in epileptic seizures. Neurochem Int 2011; 59(2): 122-137.

21. Lossi L, Castagna C, Merighi A. Caspase-3 Mediated Cell Death in the Normal Development of the Mammalian Cerebellum. Int J Mol Sci 2018; 19(12): 3999.

22. Węsierska-Gądek J, Gueorguieva M, Wojciechowski J, Tudzarova-Trajkovska S. In vivo activated caspase-3 cleaves PARP-1 in rat liver after administration of the hepatocarcinogen N-nitrosomorpholine (NNM) generating the $85 \mathrm{kDa}$ fragment. J Cell Biochem 2004; 93(4): 774-787. 